\title{
The Influence of Orbital Rotation on the Energy of Closed-Shell Wavefunctions
}

Peter A. Limacher ${ }^{a}$, Taewon D. Kim ${ }^{a}$, Paul W. Ayers ${ }^{a *}$, Paul A. Johnson ${ }^{a}$, Stijn De Baerdemacker $^{b}$, Dimitri Van Neck ${ }^{b}$, Patrick Bultinck ${ }^{c}$

${ }^{a}$ Department of Chemistry and Chemical Biology

McMaster University

Hamilton, Ontario L8S 4M1

Canada

${ }^{b}$ Center for Molecular Modelling

Ghent University

9052 Zwijnaarde

Belgium

${ }^{c}$ Department of Inorganic and Physical Chemistry

Ghent University

9000 Gent

Belgium

Keywords: doubly-occupied configuration interaction, orbital optimization, closed-shell wavefunction, static electron correlation

December 3, 2013 


\section{Abstract}

The orbital dependence of closed-shell wavefunction energies is investigated by performing doublyoccupied configuration interaction (DOCI) calculations, representing the most general class of these wavefunctions. Different local minima are examined for planar hydrogen clusters containing two, four, and six electrons applying (spin) symmetry-broken restricted, unrestricted and generalized orbitals with real and complex coefficients. Contrary to Hartree-Fock (HF), restricted DOCI is found to

properly break bonds and thus unrestricted orbitals, while providing a quantitative improvement of the energy, are not needed to enforce a qualitatively correct bond dissociation. For the beryllium atom and the $\mathrm{BH}$ diatomic, the lowest possible $\mathrm{HF}$ energy requests symmetry-broken generalized orbitals, whereas accurate results for DOCI can be obtained within a restricted formalism. Complex orbital coefficients are shown to increase the accuracy of HF and DOCI results in certain cases. The computationally inexpensive AP1roG geminal wavefunction is proven to agree very well with all DOCI results of this study. 


\section{Introduction}

There are many ways how the energy of a quantum chemical calculation can be improved by hierarchically refining the Hartree-Fock (HF) wavefunction towards the full configuration interaction (CI) limit. Besides the very popular single-reference post-HF methods such as many-body perturbation theory or the coupled-cluster approximation, there is also the realm of multireference wavefunctions that include more and more determinants, be it as a truncated CI or in the form of a complete active space calculation, to reach full CI.[1] Whereas the former family of methods rely on a reference determinant and hence traditionally engage canonical orbitals as obtained by an initial HF calculation, the energy of the latter wavefunctions is known to be orbital dependent. For this reason, the orbitals of a multireference method are usually optimized together with the expansion coefficients of the wavefunction in a multiconfiguration self-consistent field (MCSCF) procedure.[2]

One particular type of multireference method are wavefunctions which employ only closed-shell configurations in the determinantal expansion. [3-6] The most general closed-shell wavefunction is obtained by performing a doubly-occupied configuration interaction (DOCI) calculation. [7] The number

of expansion coefficients drops from $\left(\begin{array}{c}2 m \\ n\end{array}\right)$ for full CI to $\left(\begin{array}{c}m \\ n / 2\end{array}\right)$ for DOCI, where $m$ and $n$ denote the number of spatial orbitals and electrons, respectively. When these coefficients are determined variationally, the resulting DOCI energy is a lower bound to all other normalized closed-shell wavefunction energies that use the same orbitals.

DOCI was thoroughly studied in the pioneering days of quantum chemistry.[7-10] Later on, the method was abandoned due to its factorial scaling until it recently experienced a revival for various reasons, be it as a benchmark for other closed-shell methods or as a starting point for even more general wavefunctions. $[6,11-15]$ It was realized that DOCI is a particular rung on the ladder leading from HF to full CI: it is the most elaborate closed-shell wavefunction possible, and at the same time also the lowest level in a hierarchical concept called seniority, which stepwise leads to full CI. The seniority of a wavefunction is defined as the highest number of unpaired electrons that appear in the determinantal expansion.[12] In this hierarchy, DOCI is a seniority zero wavefunction and can be completed to full CI by gradually including determinants with maximally two unpaired electrons 
(seniority two), then four (seniority four) etc. until full CI is reached. It should be noted that the seniority hierarchy is not size-extensive and hence concepts from post-HF methods should be borrowed to design size-consistent extensions to DOCI. Although seniorities greater than zero are not size-extensive, DOCI itself is, just like HF is size-extensive whereas its generalization to, say CISD, is not.

DOCI, like all other closed-shell methods, is orbital dependent since all open-shell determinants are neglected and optimal orbitals need to be found along with optimal expansion coefficients to fully minimize the energy. Unlike HF, the orbital optimization for DOCI is plagued by a factorial number of local minima, many of which are close in energy. This is a general problem of MCSCF, but often is negligible in practice since the minimum found with a reasonably chosen initial orbital guess is usually very close in energy to the global minimum.[16-19] In the present study, we analyze the different minima found for DOCI when the orbitals of small hydrogen clusters and other systems are optimized variationally. Such hydrogen model systems are frequently used to address similar questions.[20, 21]

Furthermore, we investigate the changes introduced, when the orbitals are allowed to break the symmetry of the Hamiltonian.[22, 23] For HF, it is well-established that a violation of the spin operators $S^{2}$ and $S_{z}$, or the complex conjugation operator, diversifying HF into a restricted (RHF), unrestricted (UHF), or generalized (GHF) variant with real or complex orbital coefficients, lead to improved energies for certain molecules.[24, 25] The same symmetry-breaking cascade can be applied to any other type of closed-shell wavefunction giving rise to the terms RDOCI, UDOCI and GDOCI. For RDOCI, the spatial parts of $\alpha$ - and $\beta$-orbitals are identical and the electron pairs must reside within the same spatial orbital. (An alternative way would be to allow also other pairing patterns, which would correspond to restricted open-shell HF theory in some sense.) Allowing $\alpha$ - and $\beta$-orbitals to have different spatial shapes (but still requesting each electron pair to consist of an $\alpha$ - and a $\beta$-electron) breaks the $S^{2}$ symmetry and leads to the UDOCI method. Finally, GDOCI as the most general case engages fully flexible orbitals of mixed spin-character (as linear combinations of $\alpha$ - 
and $\beta$-orbitals) breaking also the $S_{z}$ symmetry. RDOCI, UDOCI and GDOCI in their real and complex implementations are studied here for the beryllium atom, the $\mathrm{BH}$ diatomic and the hydrogen clusters.

DOCI, like full CI, is a method whose computational cost grows factorially with system size and thus never will be affordable for molecules containing more than a few dozens of active electrons. Hence one might wonder why this method deserves such a detailed examination. The reason is that a recently proposed geminal wavefunction method, termed AP1roG, has shown results virtually indistinguishable from DOCI in all the cases investigated so far.[14] AP1roG is size-consistent and scales quartically with the number of electrons, such that it has all prerequisites to be applied to large systems, in particular strongly correlated materials, where single-reference methods generally fail. For this however, the effects of orbital rotation for closed-shell wavefunctions have to be understood. Due to the near equivalence of DOCI and AP1roG energies, we have chosen to present all the results only for DOCI and to summarize the errors between both methods in a final section.

The numerical calculations of this study all employ an atomic natural orbital (ANO) basis set.[26] The orbital optimization was done by a stochastic random-walk algorithm with a strict energy lowering acceptance criterion. The Hamiltonian matrix elements and molecular integrals, as well as the full CI reference results were taken from calculations with the DALTON quantum chemistry package.[27]

\section{Two-electron systems}

To start, we briefly consider two-electron systems, prototypically represented by the hydrogen molecule. Its dissociation into two infinitely separated hydrogen atoms is the textbook example par excellence for the failure of RHF.[28] Here, the only way to distribute the two electrons equally between both hydrogens is to rotate the localized 1s atomic orbitals to symmetry obedient positive and negative linear-combinations $\left(\sigma\right.$ and $\left.\sigma^{*}\right)$. Since within such orbitals the electrons repel each other even at infinite bond separation, the RHF energy can never be as low as twice the energy of a single hydrogen atom. 
The more intuitive choice of having localized 1s orbitals to represent remote atoms with no meaningful interactions left between them, is prohibited by the restriction of the method to always put two electrons (one of $\alpha$ - and one of $\beta$-spin) into the same spatial orbital. Thus, using localized orbitals results in calculating the energy of $\mathrm{H}^{+}$and $\mathrm{H}^{-}$instead, which eventually coincides with the RHF energy of delocalized orbitals at infinite distance.

In UHF, $\alpha$ - and $\beta$-electrons are allowed to occupy different spatial orbitals, which are optimized individually such that the resulting energy is in any case lower or equal to RHF. For $\mathrm{H}_{2}$, this means that after an initial region near equilibrium, which is properly described by restricted $\sigma$-orbitals, $\alpha$ and $\beta$-orbitals start to adopt distinct shapes, eventually localizing the $\alpha$-orbital on one hydrogen atom and the $\beta$-orbital on the other. Although the energy of UHF is guaranteed to improve over $\mathrm{RHF}$, there is a price to pay in the form of spin contamination, since determinants of unrestricted orbitals are no longer eigenfunctions of the $S^{2}$ operator. For converged solutions, the expectation value $\left\langle S^{2}\right\rangle$ can be calculated to assess how severe the deviations from a pure spin state are. For GHF, the $S_{z}$ symmetry is also broken as these generalized orbitals do not clearly distinguish between $\alpha$ and $\beta$-electrons.

Unlike HF, DOCI can treat two-electron systems exactly and thus symmetry-breaking is not useful. The optimal orbitals of DOCI in this case coincide with the natural orbitals (NO) of full CI and so does the energy. This also means that the optimal DOCI orbitals of two-electron systems will be delocalized, if the NOs are. In the case of $\mathrm{H}_{2}$, the DOCI orbitals leading to the lowest energy are consequently of $\sigma$ and $\sigma^{*}$ shape.

\section{Four-electron systems}

\section{$\mathbf{H}_{4}$ square}

There are several ways how two $\mathrm{H}_{2}$ moieties can be combined to a $\mathrm{H}_{4}$ cluster. Especially the planar $\mathrm{H}_{4}$ structures have been extensively studied in the literature.[20, 21, 29, 30] The $D_{4 h}$ structure deserves particular attention. This conformation is a very simple antiaromatic system, characterized by 
two degenerate NOs exactly at the Fermi level. Assuming a square of length 2 a.u. and a minimal ANO basis, the full CI energy evaluates to -1.9877 a.u. and the NOs take symmetry adapted shapes with occupation numbers $\mathrm{A}_{g}: 1.944, \mathrm{~B}_{2 u}: 1.000, \mathrm{~B}_{3 u}: 1.000$ and $\mathrm{B}_{1 g}: 0.056$. If a RDOCI is performed, there is a number of stationary points for which the orbital rotation gradient is zero, as outlined in Figure 1, none of which exactly match the full CI occupation numbers. If real orbital coefficients are used, three minima are found (orbital sets $\mathbf{V}, \mathbf{C}$ and DC) of which the vertical solution $\mathbf{V}$ is the global minimum, which also exists in a horizontal version (orbitals rotated by 90 degrees). For DOCI, the canonical HF orbitals correspond only to a local minimum indicating that the spatial symmetry must be broken in order to find a lower energy solution. The tendency of DOCI towards localized orbitals was already observed in an earlier study.[14] If complex orbitals are used, the same stationary points are found, but some local minima and maxima disappear. For example, the DC solution is now a saddle point since the imaginary distortion towards the canonical $\mathbf{C}$ solution lowers the energy. In general, the use of complex orbital coefficients is encouraged because, if not reducing the number of local minima, it smooths at least the optimization surface, such that orbital convergence is facilitated. For RHF, complex orbitals even lower the single-determinant energy from -1.8480 a.u. to -1.8585 a.u.. Such an improvement of the ground-state energy by virtue of complex orbitals is neither found for UHF or GHF, nor for any of the DOCI variants.

Considering the lowest four stationary points in Figure 1, it is apparent that they all possess the same single-determinant energy $E_{\mathrm{SD}}$, which is identical to the RHF energy with real coefficients. Since orbital rotations within the occupied and virtual orbital spaces do not affect the HF energy, all rotations between $\mathbf{C}, \mathbf{V C}, \mathbf{C V}$ and $\mathbf{V}$ leave $E_{\mathrm{SD}}$ unchanged. $E_{\mathrm{DocI}}$ on the other hand depends on these orbital rotations, as is shown in Figure 2. This is the reason that multiple minima exist for DOCI, but not for HF. The solutions $\mathbf{C}$ and $\mathbf{V}$ in RDOCI are clearly separated from each other since orbitals cannot be interchanged without crossing an energy barrier.

Proceeding to unrestricted orbitals, the number of minima for the hydrogen square does not decrease. In fact, there are three distinct minima found for UDOCI which are stable for both, real and complex orbital coefficients. The extension from UDOCI to GDOCI does not lead to any changes 
for this particular molecule, such that the same three minima found for real UDOCI orbitals persist even if complex GDOCI orbitals are applied. These minima do not possess the highly symmetric orbital shapes found for RDOCI. Instead, the orbitals are linear combinations between canonical (C) and diagonal orbitals $(\mathbf{D})$, typically two $\alpha$ - and two $\beta$-orbitals of pure state $(\mathbf{C}$ or $\mathbf{D})$ and the other four orbitals mixtures thereof $\left(x \cdot \mathbf{D C}+\sqrt{1-x^{2}} \cdot \mathbf{D}\right)$. Table 1 gives an overview of these minima and the probability to find them when optimizing a randomly chosen initial guess of complex orbitals. The global minimum, which is found in nearly all cases, still possesses two degenerate orbital pairs, one almost full, the other almost empty. In about one percent of the cases however, there is a local minimum significantly higher in energy, which has nearly the correct orbital occupation pattern with respect to full CI, yet is even higher in energy than the single-determinantal UHF method. This highlights the importance of a good initial guess for UDOCI orbitals, since otherwise an entire calculation can be trapped in a local minimum with an energy inferior to UHF. Considering $\left\langle S^{2}\right\rangle$ as a measure for spin contamination, one finds that UHF and the global minimum of UDOCI are very similar, with a slightly reduced value for the latter. On the other hand, both local minima of UDOCI, in particular the energetically unfavourable one, are more spin contaminated than UHF and hence possess more triplet character.

\section{Resizing and Distorting $\mathbf{H}_{4}$}

When hydrogen clusters are studied in literature, the interatomic distance is usually chosen to be 2 a.u., which is larger than the equilibrium structure found for $\mathrm{H}_{2}$. This is to enhance the effects of strong correlation, which are more expressed for elongated bonds. Upon variation of the side length of the square, the orbitals of a RDOCI calculation undergo a number of changes, with local minima appearing and disappearing as illustrated in Figure 3. It also shows the probability of ending up with a certain solution when randomly chosen initial orbitals are optimized by variationally minimizing the DOCI energy. Overall, the orbital set leading to the lowest energy is $\mathbf{V}$, which is also found with the highest probability at all distances. However, for small side lengths (< 1.9 a.u.) the canonical set $\mathbf{C}$ is slightly lower in energy. In the region between 2.6 a.u. 
and 3.0 a.u., the barrier between $\mathbf{V}$ and $\mathbf{C}$ disappears leaving $\mathbf{V}$ as the only minimum. After that, a short-lived minimum of reduced symmetry, similar to the UDOCI orbitals mentioned above, appears, which then unifies with the diagonal D minimum for distances $>3.5$ a.u.. At infinite separation, the D and $\mathbf{V}$ orbitals both return the correct energy of four isolated hydrogen atoms with a probability distribution of $1: 2$, since the $\mathbf{V}$ orbitals possess a vertical and a horizontal variant.

In Figure 4 the global minimum for RDOCI is compared to UDOCI, RHF, UHF, and the full CI singlet and triplet ground state energies. In the case of RHF, the use of complex orbital coefficients leads to a slight energy decrease. In all other cases, results for real and complex orbitals are identical. Furthermore, the GHF/GDOCI solutions coincide with UHF/UDOCI at all distances. In the RDOCI curve of Figure 4, the change of the minimum energy basis at 1.9 a.u. can easily be spotted as a discontinuity of the energy. A similar, yet less discernible discontinuity exists for UDOCI at 2.3 a.u. that also leads to a jump of $\left\langle S^{2}\right\rangle$. The evolution of $\left\langle S^{2}\right\rangle$ for UHF and UDOCI indicates that the dissociation into four isolated hydrogen atoms is realized as a triplet state. Consequently, both energy curves follow more closely the full CI triplet curve than the singlet curve. In the region of small interatomic distances, the energy of UDOCI (and to a lesser extent also UHF) is even lower than the singlet full CI solution clearly proofing the substantial triplet character of these unrestricted wavefunctions.

Figure 5 shows the behaviour of symmetry-broken orbitals in rectangular $\mathrm{H}_{4}$ systems. Instead of rescaling the entire $\mathrm{H}_{4}$ square, one side of the rectangle stays now fixed at 2 a.u., whereas the length of the other side is variable. Such a distortion allows the orbitals to form energetically preferred $\mathrm{H}_{2}$ bonds in either horizontal or vertical direction, depending on the relative magnitude of the fixed and variable sides of the rectangle. Due to these designated $\mathrm{H}_{2}$ fragments within $\mathrm{H}_{4}$, the results obtained with restricted orbitals improve and make unrestricted orbitals only necessary in the region of nearly equal bond lengths, as the curves for HF and DOCI in Figure 5 illustrate. However, the range of this region is larger for HF, such that the overall need of unrestricted orbitals is less pronounced for DOCI. The aforementioned energy improvement of complex RHF over real RHF orbitals exists only for a very narrow region at the near square geometry $(2 \pm 0.05$ a.u. $)$ and completely disappears for 


\section{RDOCI.}

Another way of distorting the $\mathrm{H}_{4}$ square is to shear two opposite sides such that the four hydrogen atoms form a parallelogram of constant height.[31] Taking the prototypical square of side length 2.0 a.u., the shearing leads to parallelograms of base length and height 2.0 a.u., for which energies and $\left\langle S^{2}\right\rangle$ are reported in Figure 6. Similar to the rectangle, unrestricted orbitals improve the energies only around the square geometry and UHF is beneficial for a longer range than UDOCI. Further, the UDOCI ground state undergoes an abrupt change of state at a displacement length of 0.37 a.u., visible as a discontinuity of $\left\langle S^{2}\right\rangle$, while following the full CI energy curve very closely.

\section{Beryllium}

For all forms of $\mathrm{H}_{4}$ investigated here, the generalized GHF and GDOCI methods always coincide with UHF and UDOCI. An exemplary case of a system with a GHF energy lower than the corresponding UHF energy is the beryllium atom, at least for small basis sets.[25] Table 2 summarizes the characteristic quantities of Be obtained with different methods. For HF, the energy drops from restricted to unrestricted and finally generalized orbitals at the expense of introducing spin contamination. The reason for the lower UHF and GHF energies is the possibility to move some of the electronic charge from $s$-orbitals into $p$-orbitals, which is prohibited for RHF due to symmetry restrictions. However, this charge shift is achieved by artificially breaking the spin symmetry and hence is energetically not very efficient. Note that for optimized GHF orbitals the $S_{z}$ expectation value is zero although every individual orbital has a mixed $\alpha$ and $\beta$ character.

For DOCI, the $p$-orbitals naturally can be populated simply by including determinants with $p$ orbitals in the CI expansion. It is therefore not necessary to break spin symmetry at all, and neither symmetry-broken orbitals nor the use of complex coefficients leads to any changes of the energy obtained with real RDOCI orbitals. It is thus sufficient to use restricted orbitals for such problems. In fact, beryllium as a quasi two-electron system (due to the energetically low-lying core electrons) is modelled nearly perfectly by DOCI in comparison to full CI. 


\section{Six-electron systems}

\section{$\mathbf{H}_{6}$ hexagon}

Hexagonal $\mathrm{H}_{6}$ is a frequently used toy system to model aromaticity. Unlike for the $\mathrm{H}_{4}$ square, RHF is capable to describe $\mathrm{H}_{6}$ at short distances, since the distinction between occupied and virtual orbitals is completely unambiguous. Assuming a hexagonal side length of 2.0 a.u., orbital optimized RDOCI finds three energy minima reported in Figure 7 . Although the $\mathbf{N}$ set strongly resembles the canonical HF orbitals (that are identical with the full CI natural orbitals due to symmetry), this is a peculiarity of the near equilibrium geometry and is different for side lengths $>2.0$ a.u. The discrepancies between both can be seen as subtle variations of the orbital occupation numbers and a single-determinant energy slightly higher than for RHF. Just like in the $\mathrm{H}_{4}$ square, the formation of localized bonding $\sigma$ and antibonding $\sigma^{*}$-orbitals leads also to a lower DOCI energy for $\mathrm{H}_{6}$. Different solutions emerge, depending on the connectivity of these localized orbital pairs. Two of them (D and $\mathbf{T})$ are found to be stable with respect to orbital optimization. Figure 8 shows the evolution of the $\mathbf{N}, \mathbf{D}$ and $\mathbf{T}$ minima when the size of the hexagon is changed. For short bonds, $\mathbf{N}$ is the global RDOCI minimum. It is then replaced by $\mathbf{T}$, for distances $>1.7$ a.u. up to complete dissociation.

Contrary to the antiaromatic $\mathrm{H}_{4}$ square, unrestricted orbitals not always lead to lower energies for $\mathrm{H}_{6}$. For side lengths between 1.9 a.u. and 2.6 a.u. UDOCI coincides with the $\mathbf{T}$ orbitals of RDOCI. For larger values, UDOCI provides a solution lower in energy than RDOCI. Both eventually coincide at infinite length. For compressed $\mathrm{H}_{6}$, there appears again a lower UDOCI solution that only exists using complex orbital coefficients. From Figure 9 can be seen that for UHF, no such energy lowering is possible at short side lengths, so that real and complex coefficients provide identical solutions for RHF and UHF over the entire dissociation range. The complex UDOCI energy curve evolves largely parallel to the $\mathbf{N}$ orbital set and thus disappears, once the real, restricted $\mathbf{T}$ solution is energetically favourable (Figure 8).

The analysis of $\left\langle S^{2}\right\rangle$ in Figure 9 shows that UDOCI is again less prone to spin contamination than UHF. At infinite distances UHF approaches an $\left\langle S^{2}\right\rangle$ of 3 a.u. and UDOCI 2 a.u. respectively. 
For short distance $\mathrm{H}_{6}$, spin contamination is very small for the complex UDOCI solution, but the change of orbitals at 1.9 a.u. is still clearly visible as a discontinuity of $\left\langle S^{2}\right\rangle$.

Table 3 shows characteristic points on the energy curves in Figure 9. Besides the side length $r_{\min }$ that leads to the minimal energy of the hexagonal $\mathrm{H}_{6}$ structure, there is also the critical radius $r_{\mathrm{c}}$ reported, at which a distortion into pairs of hydrogen (forming $\left(\mathrm{H}_{2}\right)_{3}$ while retaining each atom's distance to the center) is energetically favourable. Hexagons with $r<r_{\mathrm{c}}$ prefer $D_{6 h}$ symmetry, whereas for $r>r_{\mathrm{c}}$ dimerization occurs. From the orbital symmetries of Figure 7 , it is clear that only the $\mathbf{N}$ and $\mathbf{D}$ orbitals can possess the hexagonal structure as a stationary point, whereas the T orbitals have a cusp there (created by the two different potential energy curves for either way of pairing neighboring $\mathrm{H}$ atoms, each having a nonzero slope at the hexagonal structure). The $\mathbf{T}$ orbitals themselves thus never predict any $D_{6 h}$ structure to be stable. However, at small enough distances, the transformation of $\mathbf{T}$ into $\mathbf{N}$ is not only energetically favourable, but also possible without passing an energy barrier such that $D_{6 h}$ is the preferred geometry at fixed small radii.

\section{BH dissociation}

The dissociation of $\mathrm{BH}$ into two isolated atoms of boron and hydrogen is another case in which the application of generalized orbitals with mixed $\alpha$ and $\beta$ character leads to improved HF energies. [25] The problem with RHF is, analogous to Be in Table 2, that due to symmetry restrictions all electrons must occupy the rotationally invariant $\sigma$-orbitals, although some gain in energy would be possible by populating also the $\pi$-orbitals. The breaking of spin symmetry allows exactly that and leads to improved UHF and GHF results, as illustrated in Figure 10. In contrast to the isolated Be atom, in $\mathrm{BH}$ there is also simultaneous bond breaking, which requests the UHF functionality too, such that after a distance of 3.5 a.u. UHF and GHF coincide and the wavefunction changes character afterwards. This can be spotted noticing the abrupt changes of $\left\langle S^{2}\right\rangle$ and the drop of the electron density in the $\pi$-orbitals to zero. RDOCI on the other hand all by itself correctly describes bond breaking as well as the non-zero density in the $\pi$-orbitals. Consequently, the solutions for symmetrybroken UDOCI and GDOCI orbitals converge to a RDOCI solution and symmetry-breaking is never 
necessary for BH over the entire range of bond lengths. Furthermore, the RDOCI energy is constantly very close to full CI.

\section{Geminal wavefunctions}

The AP1roG wavefunction is an antisymmetric product of non-orthogonal geminals.[14] The energies obtained with this method usually differ very little from DOCI energies, such that on the scale of the presented figures, no deviation could be spotted. Instead, the energy differences between AP1roG and DOCI are summarized in Table 4. It is found that even the largest deviations stay in the submilli-Hartree regime. They typically occur in the region of bond breaking, where the multireference character of the wavefunction is particularly strong. But even in the worst case $\left(\mathrm{H}_{6}\right.$ dissociation), the maximal difference is only about half a milli-Hartree. Overall, the AP1roG wavefunction is certainly capable of mimicking DOCI energies in great detail.

The excellent agreement between DOCI and AP1roG energies is only observed when the set of orbitals correspond to a local energy minimum. In Figure 11, the energy difference between the DOCI and AP1roG method is given for the $\mathrm{H}_{4}$ square as a function of the same orbital rotation parameters as in Figure 2. Clearly visible, the close match of the energies is lifted already by small distortions from a local orbital minimum. Although DOCI and AP1roG share the same stationary points, the saddle points CV and VC do not have such an energy coalescence. The energy difference for these points lies around 0.0002 a.u. which is about ten times higher (and of opposite sign) than $\Delta E$ for $\mathrm{V}$. Note that due to orbital symmetry, $\Delta E$ is exactly zero for $\mathrm{C}$.

Although the agreement of DOCI and AP1roG is generally the best for orbitals representing local minima, $\Delta E$ stays small enough on the entire orbital rotation surface, such that a direct optimization of AP1roG orbitals is a straightforward task (instead of taking the orbitals from a DOCI calculation, as it is done in this study). This is a necessary requirement if AP1roG should become a practical stand-alone method.

When optimizing AP1roG orbitals directly, allowing the orbital coefficients to be 
complex numbers, one consideration is to run into problems with complex energy solutions due to the non-hermiticity of the projected Schrödinger equation. This is, in fact, a known problem in coupled-cluster theory and complex energy solutions are possible even for purely real orbitals.[32] Since AP1roG is a special case of coupled-cluster with only pair amplitudes different from zero,[14] one should not encounter any new type of problem that are not already present in coupled-cluster theory. Within this study, all the AP1roG energies obtained with complex orbitals were purely real. Another benefit of AP1roG being a particular coupled-cluster method, is the ease of developing a stable and efficient numerical orbital optimizer for AP1roG, since all the techniques of orbital-optimized coupled-cluster theory can be applied in a straightforward manner.[1] This will be the topic of a future publication.

\section{Conclusions}

The DOCI approach, as the best possible closed-shell wavefunction, and likewise AP1roG, as a computationally inexpensive, yet very accurate approximation to DOCI, overcome many drawbacks of single-reference methods. In particular, these methods are able to account for strong electron correlation and thus can predict bond separation reactions with great accuracy. The main disadvantages are their orbital dependence and the possibility that a variational optimization of the orbitals leads to a local energy minimum. Multiple solutions exist already for a minimal basis set $\mathrm{H}_{4}$ square molecule. As a remedy, localized orbitals should be used as initial guesses for an orbital optimization procedure, since the global energy minimum is usually characterized by orbitals localized on maximally two atoms (forming bonding and antibonding orbital pairs). Only for compressed molecular structures, the delocalized canonical orbitals possess a lower energy solution, which normally does not differ very much from the energy obtained with paired orbitals. The existence of an optimized solution with delocalized orbitals is nevertheless an important prerequisite to retain molecular point group symmetries. It enables DOCI, and in particular also AP1roG, to properly describe the symmetry of molecules like benzene. 
Considering symmetry-broken orbitals, the need for an unrestricted formulation of DOCI is less urgent than for HF. Since RDOCI describes bond dissociation qualitatively correct already, its generalization to UDOCI only leads to improved energies if several equivalent pairing schemes are possible $\left(\mathrm{H}_{4}\right.$ square, $\mathrm{H}_{6}$ hexagon). Another reason in $\mathrm{HF}$ to use unrestricted (and even generalized) orbitals is the capability of UHF and GHF to populate the orbitals of different spatial symmetries with fractional numbers, whereas RHF enforces integer sums for every symmetry block. Such considerations are completely void for DOCI, and consequently the energy of the Be atom and the BH dissociation curve are predicted completely satisfactorily with RDOCI. For all systems studied here, GDOCI as the most generalized DOCI approach never leads to a lowering of the energy, and can always be recast as UDOCI.

Finally, the merits of complex orbitals should be highlighted. In two cases, namely the $\mathrm{H}_{4}$ square RHF and the $\mathrm{H}_{6}$ UDOCI energy, solutions with complex orbitals lead to lower energies than it is possible for real coefficients. But also in systems where the energy does not alter with the introduction of complex orbitals, it is still advantageous to use them because of the smoother energy surfaces and the facilitated convergence of the orbital optimization procedure.

\section{Acknowledgments}

This research was supported by the Swiss National Science Foundation fellowship PBEZP2-140249 and by NSERC. PAJ acknowledges a Vanier Canada Graduate scholarship. SDB, DVN and PB are members of the Ghent-Brussels Quantum Chemistry and Molecular Modeling alliance. The authors acknowledge a generous allocation of computer time granted by SHARCNET, a partner consortium in the Compute Canada national HPC platform. 


\section{References}

[1] T. Helgaker, P. Jørgensen, and J. Olsen, Molecular Electronic-Structure Theory, Wiley, Chichester, 2000.

[2] F. Jensen, Introduction to Computational Chemistry, John Wiley \& Sons, New York, 2007.

[3] D. Cook, Mol. Phys. 30, 733 (1975).

[4] D. D. Shillady, S. Cutler, L. F. Jones, and L. B. Kier, Int. J. Quantum Chem. 38, 153 (1990).

[5] M. Couty and M. B. Hall, J. Phys. Chem. A 101, 6936 (1997).

[6] C. Kollmar, J. Chem. Phys. 121, 11581 (2004).

[7] F. Weinhold and E. B. Wilson, J. Chem. Phys. 46, 2752 (1967).

[8] T. L. Allen and H. Shull, J. Phys. Chem. 66, 2281 (1962).

[9] D. W. Smith and S. J. Fogel, J. Chem. Phys. 43, S91 (1965).

[10] A. Veillard and E. Clementi, Theor. Chim. Acta 7, 133 (1967).

[11] C. Kollmar and B. A. Heß, J. Chem. Phys. 119, 4655 (2003).

[12] L. Bytautas, T. M. Henderson, C. A. Jiménez-Hoyos, J. K. Ellis, and G. E. Scuseria, J. Chem. Phys. 135, 044119 (2011).

[13] P. A. Johnson, P. W. Ayers, P. A. Limacher, S. De Baerdemacker, D. Van Neck, and P. Bultinck, Comput. Theor. Chem. 1003, 101 (2013).

[14] P. A. Limacher, P. W. Ayers, P. A. Johnson, S. De Baerdemacker, D. Van Neck, and P. Bultinck, J. Chem. Theory Comput. 9, 1394 (2013).

[15] P. A. Limacher, P. W. Ayers, P. A. Johnson, S. De Baerdemacker, D. Van Neck, and P. Bultinck, Phys. Chem. Chem. Phys. submitted. 
[16] C. Angeli, C. J. Calzado, R. Cimiraglia, S. Evangelisti, and D. Mayna, Mol. Phys. 101, 1937 (2003).

[17] M. Lewin, Arch. Rational Mech. Anal. 171, 83 (2004).

[18] E. Cancès, H. Galicher, and M. Lewin, J. Comput. Phys. 212, 73 (2006).

[19] M. Lewin, J. Math. Chem. 44, 967 (2008).

[20] J. B. Robinson and P. J. Knowles, J. Chem. Phys. 137, 054301 (2012).

[21] J. K. Ellis, R. L. Martin, and G. E. Scuseria, J. Chem. Theory Comput. 9, 2857 (2013).

[22] G. E. Scuseria, C. A. Jiménez-Hoyos, T. M. Henderson, K. Samanta, and J. K. Ellis, J. Chem. Phys. 135, 124108 (2011).

[23] C. A. Jiménez-Hoyos, T. M. Henderson, and G. E. Scuseria, J. Chem. Theory Comput. 7, 2667 (2011).

[24] P.-O. Löwdin and I. Mayer, Adv. Quantum Chem. 24, 79 (1992).

[25] J. L. Stuber and J. Paldus, Symmetry breaking in the independent particle model, in Fundamental World of Quantum Chemistry: A Tribute to the Memory of Per-Olov Löwdin, edited by P. Löwdin, E. Brändas, and E. Kryachko, volume 1, pages 67-139, Kluwer Academic Publishers, Dordrecht, 2003.

[26] P.-O. Widmark, P.-A. Malmqvist, and B. O. Roos, Theor. Chim. Acta 77, 291 (1990).

[27] DALTON, a molecular electronic structure program, Release 2.0 (2005), see http://www.kjemi . uio.no/software/dalton/dalton.html., 2005.

[28] A. Szabo and N. S. Ostlund, Modern Quantum Chemistry: Introduction to Advanced Electronic Structure Theory, Macmillan, New York, 1982.

[29] J. Paldus, P. Piecuch, L. Pylypow, and B. Jeziorski, Phys. Rev. A 47, 2738 (1993). 
[30] K. Kowalski and K. Jankowski, Phys. Rev. Lett. 81, 1195 (1998).

[31] E. Neuscamman, CoRR arXiv:1308.5297 (2013).

[32] J. Paldus, M. Takahashi, and R. W. H. Cho, Phys. Rev. B 30, 4267 (1984). 


\section{Tables}

Table 1. Unrestricted DOCI energies of the three minimal energy orbital sets for the $\mathrm{H}_{4}$ square, orbital occupation numbers $\left(n_{i}\right)$ and expectation value $\left\langle S^{2}\right\rangle$, as well as the probability of finding each minimum when starting the optimization with random complex orbitals (based on 10'000 calculations).

\begin{tabular}{cccccccc}
\hline \hline Method & $E /$ a.u. & $n_{1}$ & $n_{2}$ & $n_{3}$ & $n_{4}$ & $\left\langle S^{2}\right\rangle /$ a.u. & Probability \\
\hline UDOCI & -1.9767 & 1.988 & 1.988 & 0.012 & 0.012 & 1.053 & $98.89 \%$ \\
& -1.9664 & 1.998 & 1.988 & 0.012 & 0.002 & 1.155 & $0.25 \%$ \\
& -1.9406 & 1.997 & 1.000 & 1.000 & 0.003 & 1.500 & $0.85 \%$ \\
\hline UHF & -1.9637 & 2.000 & 2.000 & 0.000 & 0.000 & 1.085 & $100 \%$ \\
\hline \hline
\end{tabular}


Table 2. Energy, total electron density in all $p$-orbitals and spin expectation values $\left\langle S^{2}\right\rangle$ and $\left\langle S_{z}\right\rangle$ for the beryllium atom obtained with complex restricted, unrestricted and generalized optimized orbitals using an $\operatorname{ANO}(3 \mathrm{~s} 1 \mathrm{p})$ basis set.

\begin{tabular}{ccccc}
\hline \hline Method & $E /$ a.u. & $\sum n_{p}$ & $\left\langle S^{2}\right\rangle /$ a.u. & $\left\langle S_{z}\right\rangle /$ a.u. \\
\hline full CI & -14.617571 & 0.19597 & & \\
RHF & -14.572837 & 0.00000 & & \\
UHF & -14.573161 & 0.06387 & 0.1237 & \\
GHF & -14.573190 & 0.06953 & 0.1358 & 0.0000 \\
RDOCI & -14.617515 & 0.19595 & & \\
UDOCI & -14.617515 & 0.19595 & 0.0000 & \\
GDOCI & -14.617515 & 0.19595 & 0.0000 & 0.0000 \\
\hline \hline
\end{tabular}


Table 3. Side length $r$ and corresponding energies $E$ for characteristic points of a minimal basis set $\mathrm{H}_{6}$ hexagon. Reported are the minimum of the potential energy curve $\left(r_{\min }\right.$ and $\left.E_{\min }\right)$, as shown in Figure 9, and the critical side length $\left(r_{\mathrm{c}}\right.$ and $\left.E_{\mathrm{c}}\right)$, after which the $D_{6 h}$ molecular point group symmetry is unstable with respect to $D_{3 h}$ (hydrogen dimerization).

\begin{tabular}{ccccc}
\hline \hline Method & $r_{\min } /$ a.u. & $E_{\min } /$ a.u. & $r_{\mathrm{c}} /$ a.u. & $E_{\mathrm{c}} /$ a.u. \\
\hline FCI & 2.004 & -3.2793 & 1.627 & -3.1934 \\
HF & 1.941 & -3.2043 & 1.584 & -3.1112 \\
RDOCI & 1.981 & -3.2438 & 1.624 & -3.1634 \\
UDOCI & 1.981 & -3.2438 & 1.652 & -3.1816 \\
\hline \hline
\end{tabular}


Table 4. Deviation of the AP1roG geminal method from DOCI. The error $\left(\Delta E=E_{\mathrm{AP} 1 \mathrm{roG}}-E_{\mathrm{DOCI}}\right)$ is summarized by the root-mean-square deviation over the range reported in the corresponding figures, the largest overestimation $\left(\Delta E_{\max }\right)$ of DOCI by AP1roG, and the largest underestimation $\left(\Delta E_{\min }\right)$ together with the distances, at which these occur. Energies are given in milli-Hartree, distances in Bohr.

\begin{tabular}{cccccccc}
\hline \hline System & Method & Ref. & $E_{\text {rmsd }}$ & $r_{\max }$ & $\Delta E_{\max }$ & $r_{\min }$ & $\Delta E_{\min }$ \\
\hline $\mathrm{H}_{4}$ square & RAP1roG & Fig. 4 & 0.0833 & 1.89 & 0.000 & 3.62 & -0.132 \\
$\mathrm{H}_{4}$ square & UAP1roG & Fig. 4 & 0.1225 & 2.34 & 0.035 & 3.92 & -0.210 \\
$\mathrm{H}_{4}$ rect. & RAP1roG & Fig. 5 & 0.0084 & 0.00 & 0.000 & 2.00 & -0.021 \\
$\mathrm{H}_{4}$ rect. & UAP1roG & Fig. 5 & 0.0086 & 2.00 & 0.020 & 2.25 & -0.024 \\
$\mathrm{H}_{4}$ para. & RAP1roG & Fig. 6 & 0.0185 & 1.04 & 0.027 & 0.00 & -0.021 \\
$\mathrm{H}_{4}$ para. & UAP1roG & Fig. 6 & 0.0195 & 1.04 & 0.027 & 0.63 & -0.021 \\
$\mathrm{H}_{6}$ hex. & RAP1roG & Fig. 9 & 0.2570 & 2.36 & 0.039 & 3.63 & -0.514 \\
$\mathrm{H}_{6}$ hex. & UAP1roG & Fig. 9 & 0.2747 & 1.96 & 0.066 & 3.75 & -0.495 \\
BH & RAP1roG & Fig. 10 & 0.1717 & 5.43 & 0.250 & 1.02 & 0.040 \\
\hline \hline
\end{tabular}




\section{Figures}
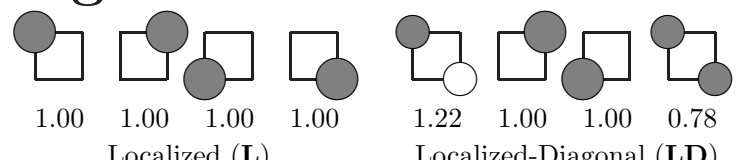

$\begin{array}{llll}1.22 & 1.00 & 1.00 & 0.78\end{array}$

Localized-Diagonal (LD)

Global maximum in $\mathbb{R}$ and $\mathbb{C}$ Saddle point in $\mathbb{R}$ and $\mathbb{C}$

$E_{\text {DOCI }}=-0.7583$ a.u. $\quad E_{\text {DOCI }}=-0.8332$ a.u.

$E_{\mathrm{SD}}=-0.7569$ a.u. $\quad E_{\mathrm{SD}}=-0.6339$ a.u.

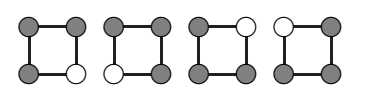

$\begin{array}{llll}1.00 & 1.00 & 1.00 & 1.00\end{array}$

Asymmetric (A)

Local maximum in $\mathbb{R}$

Saddle point in $\mathbb{C}$

$E_{\mathrm{DOCI}}=-1.1674$ a.u.

$E_{\mathrm{SD}}=-0.9159$ a.u.

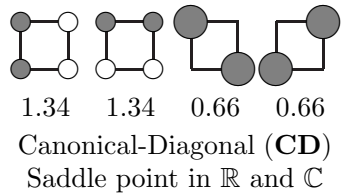

$E_{\text {DOCI }}=-1.1808$ a.u.

$E_{\mathrm{SD}}=-0.9384 \mathrm{a} . \mathrm{u}$

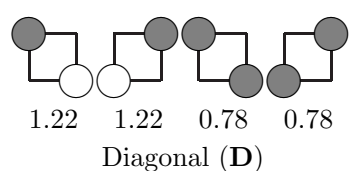

Saddle point in $\mathbb{R}$ and $\mathbb{C}$

$$
\begin{aligned}
E_{\mathrm{DOCI}} & =-1.3350 \mathrm{a} . \mathrm{u} . \\
E_{\mathrm{SD}} & =-0.9384 \mathrm{a} . \mathrm{u} .
\end{aligned}
$$

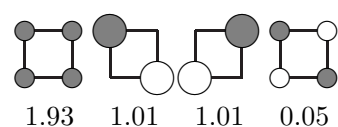

Diagonal-Canonical (DC)

Local minimum* in $\mathbb{R}$ Saddle point in $\mathbb{C}$

$E_{\text {DOCI }}=-1.8434$ a.u.

$E_{\mathrm{SD}}=-1.7894$ a.u.
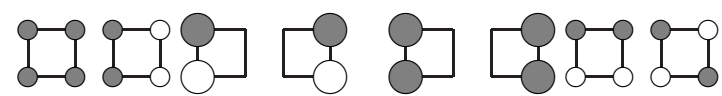

$\begin{array}{llll}1.99 & 1.93 & 0.04 & 0.04\end{array}$

Canonical-Vertical (CV)

Saddle point in $\mathbb{R}$ and $\mathbb{C}$

$E_{\mathrm{DOCI}}=-1.8845$ a.u.

$E_{\mathrm{SD}}=-1.8480$ a.u.

$\begin{array}{llll}1.95 & 1.95 & 0.08 & 0.01\end{array}$

Vertical-Canonical (VC)

Saddle point in $\mathbb{R}$ and $\mathbb{C}$

$E_{\mathrm{DOCI}}=-1.8863 \mathrm{a} . \mathrm{u}$

$E_{\mathrm{SD}}=-1.8480$ a.u.

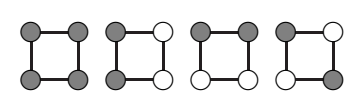

$\begin{array}{llll}2.00 & 1.00 & 1.00 & 0.00\end{array}$

Canonical $(\mathbf{C})$
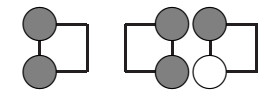

$\begin{array}{llll}1.92 & 1.92 & 0.08 & 0.08\end{array}$

Vertical (V)

Local minimum in $\mathbb{R}$ and $\mathbb{C}$ Global minimum in $\mathbb{R}$ and $\mathbb{C}$

$E_{\text {DOCI }}=-1.9196$ a.u.

$E_{\text {DOCI }}=-1.9246$ a.u.

$E_{\mathrm{SD}}=-1.8480$ a.u.

$E_{\mathrm{SD}}=-1.8480$ a.u.

Orbital weight: $1 \bigcirc \frac{1}{\sqrt{2}} \bigcirc \frac{1}{2} \bigcirc-\frac{1}{2} \bigcirc-\frac{1}{\sqrt{2}}$

*For DC, a small rotation of the degenerate diagonal orbitals does not lead to any change in the energy, hence it can be considered as a quasi-minimum in $\mathbb{R}$

Figure 1: The stationary points of the RDOCI energy with respect to orbital variation. Every diagram contains the orbital shapes, occupation numbers, characterization of the stationary point for real and complex orbital rotations, the total DOCI energy, as well as the single-determinant energy (occupying the two orbitals with highest occupation number). 


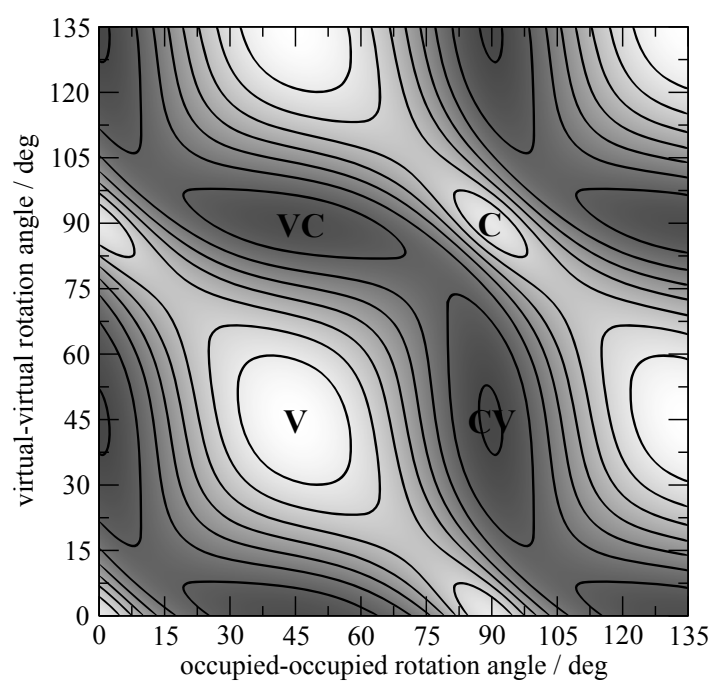

Figure 2: RDOCI energy as a function of orbital rotations between the two occupied and the two virtual canonical HF orbitals connecting the four stationary points V, C, CV and $\mathrm{VC}$ in the $\mathrm{H}_{4}$ square. The spacing between two contours corresponds to an energy change of 0.005 a.u. with a dark background indicating regions of higher energy. The pattern repeats after rotation about 90 degrees in either direction. 

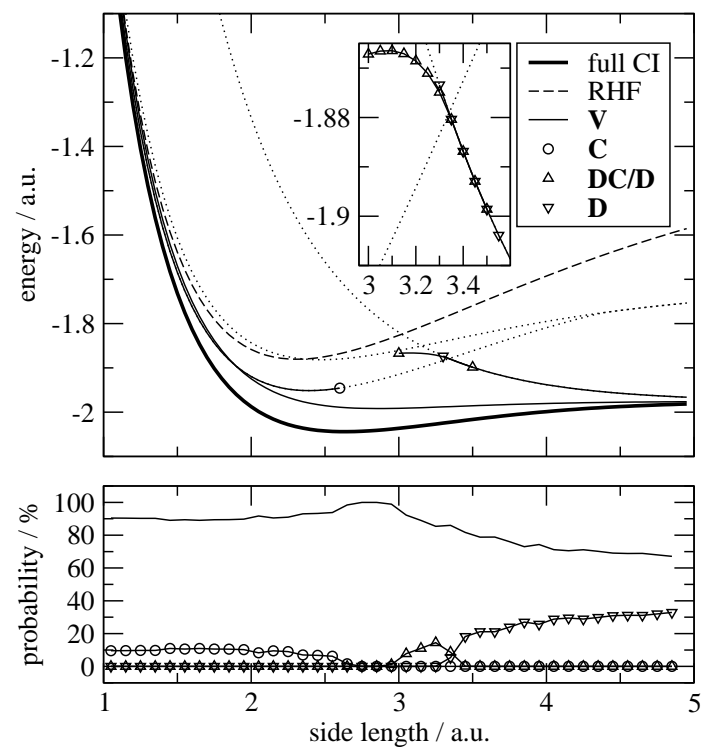

Figure 3: RDOCI energies of the $\mathrm{H}_{4}$ square for different sets of optimized orbitals as a function of the interatomic distance. The nomenclature for the orbitals is given in Figure 1. The inset is a zoom-in at the region where two local minima coalesce into one. The dotted lines represent the continuation of the $\mathbf{C}, \mathbf{D}$ and $\mathbf{D C}$ energies, where these orbital sets are no minima. The lower panel illustrates the likelihood to converge to a particular orbital minimum when the initial orbitals are chosen randomly (based on 1000 calculations per data point). 

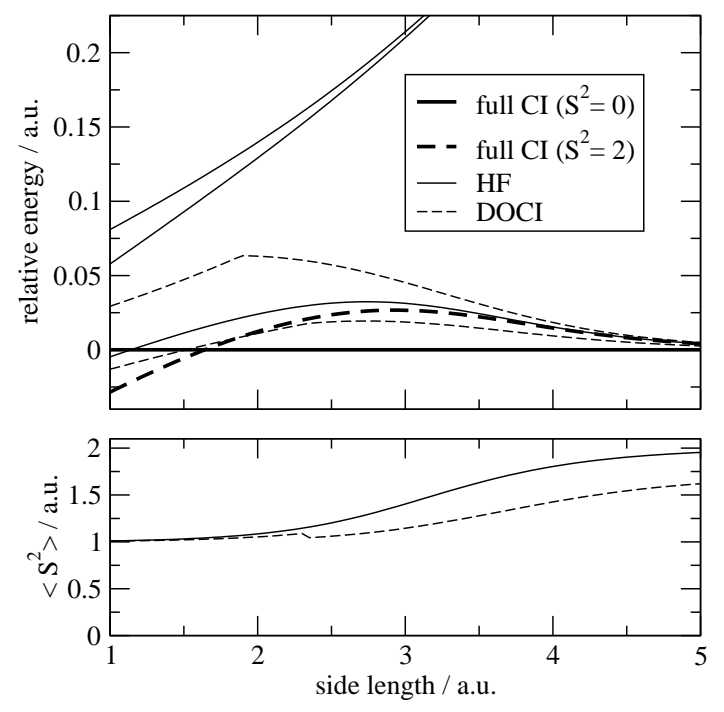

Figure 4: Energy difference from the full CI singlet energy (top) and $\left\langle S^{2}\right\rangle$ of UHF and UDOCI solutions (bottom) for minimal basis set $\mathrm{H}_{4}$ as a function of the interatomic distance. For HF, the three energy curves correspond to real RHF, complex RHF and real UHF optimized orbitals (in the order of decreasing energy). For DOCI, results for optimized real RDOCI and real UDOCI orbital coefficients are shown. 

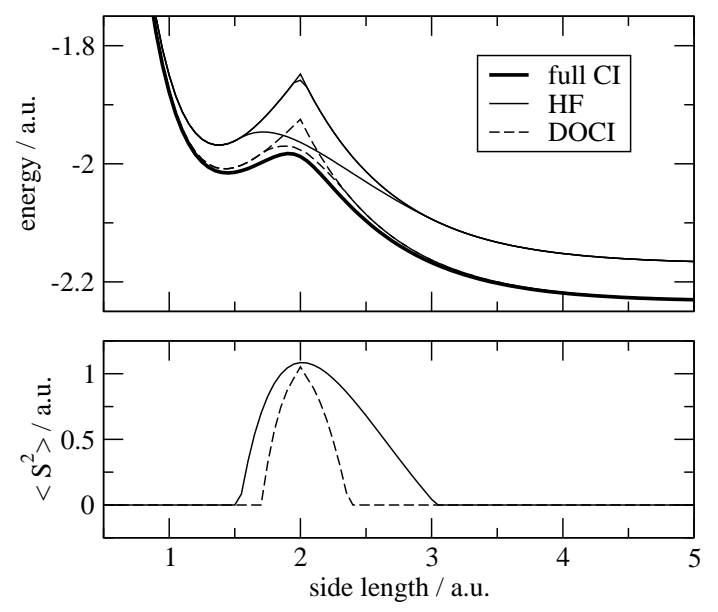

Figure 5: Energy (top) and $\left\langle S^{2}\right\rangle$ (bottom) for a minimal basis set $\mathrm{H}_{4}$ rectangle with one side length fixed at 2 a.u.. Close to the square geometry, HF diversifies into three curves for real RHF, complex RHF and real UHF orbitals (in the order of decreasing energy) whereas DOCI splits into two curves (real RDOCI and real UDOCI). 

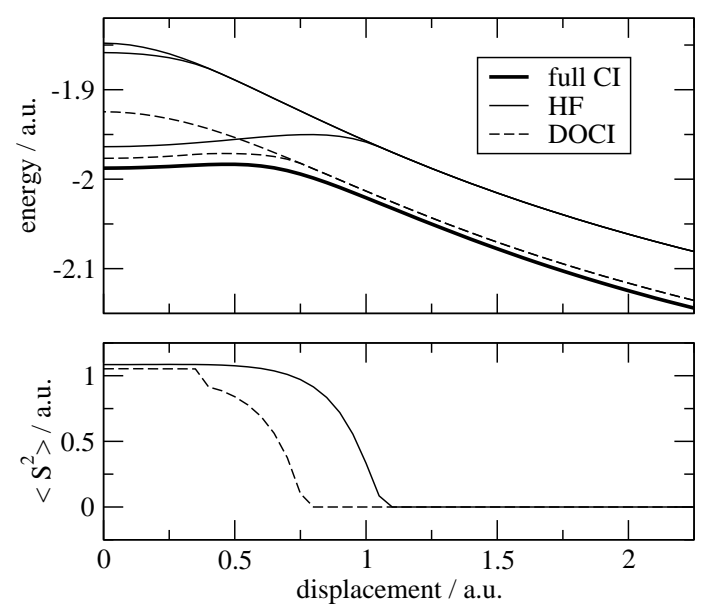

Figure 6: Energy (top) and $\left\langle S^{2}\right\rangle$ (bottom) for a minimal basis set $\mathrm{H}_{4}$ parallelogram with base length and height 2 a.u.. The same splitting of the orbitals into real RHF, complex RHF real UHF, real RDOCI and real UDOCI applies as in Figure 5. 


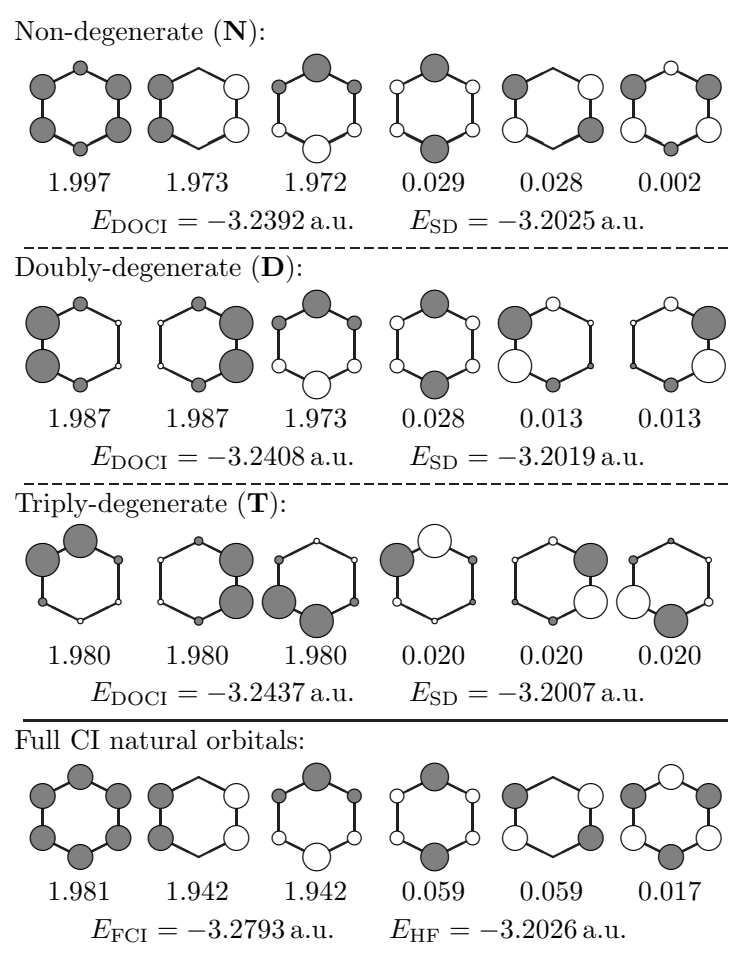

Figure 7: Schematic representation (nomenclature, shape and occupation numbers) of the three orbital sets leading to minimal RDOCI energies for the $\mathrm{H}_{6}$ hexagon with side length 2.0 a.u. applying an $\mathrm{ANO}(1 \mathrm{~s})$ basis set. On the bottom, the comparison is made to full CI NO populations and energy, as well as to the RHF energy. 


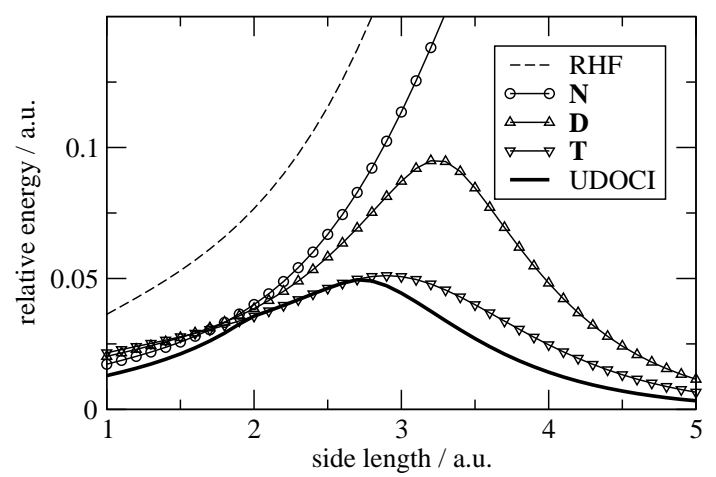

Figure 8: DOCI energies of different optimized orbitals for a minimal basis set $\mathrm{H}_{6}$ hexagon as a function of the side length. The energy is given with respect to full CI. Besides the three restricted DOCI solutions described in Figure 7, also the lowest possible DOCI energy is shown, obtained by an UDOCI calculation with complex orbital coefficients. 

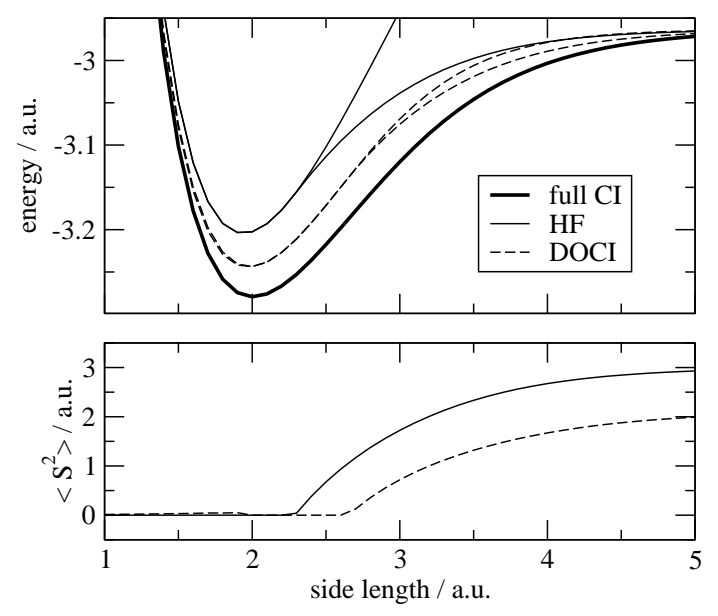

Figure 9: Energy (top) and $\left\langle S^{2}\right\rangle$ (bottom) for a minimal basis set $\mathrm{H}_{6}$ hexagon as a function of the side length. For enlarged bonds, HF and DOCI split into a restricted and an unrestricted curve. For compressed bonds, a UDOCI solution of lower energy is found when complex orbital coefficients are applied. 

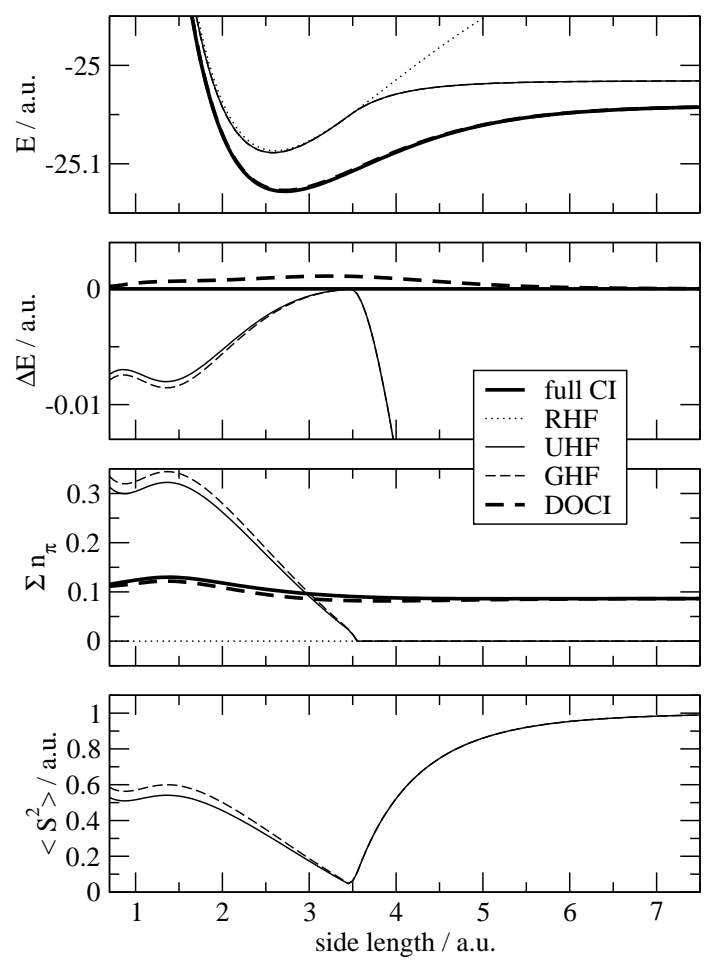

Figure 10: Energies (top), sum of the electron density in the four $\pi$-orbitals (middle) and $\left\langle S^{2}\right\rangle$ (bottom) for the $\mathrm{BH}$ bond dissociation using a minimal ANO basis set. For a better distinction of the energy curves, relative energies are also presented according to the definitions $\Delta E_{\mathrm{UHF}}=E_{\mathrm{UHF}}-E_{\mathrm{RHF}}$, $\Delta E_{\mathrm{GHF}}=E_{\mathrm{GHF}}-E_{\mathrm{RHF}}$ and $\Delta E_{\mathrm{DOCI}}=E_{\mathrm{DOCI}}-E_{\mathrm{FCI}}$. 


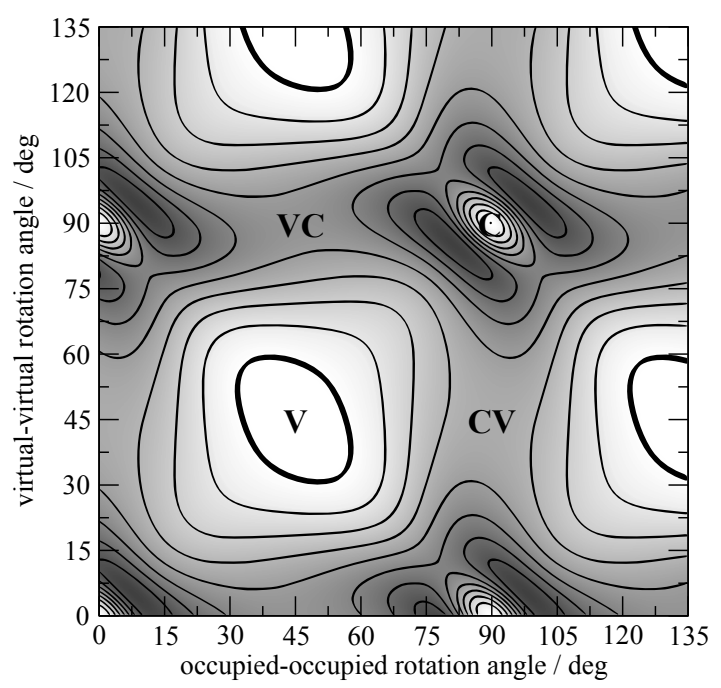

Figure 11: The error of AP1roG relative to DOCI $\left(\Delta E=E_{\mathrm{AP} 1 \mathrm{roG}}-E_{\mathrm{DOCI}}\right)$ for the $\mathbf{H}_{4}$ square with side length 2 a.u. ploted for the same orbitals as in Figure 2. The spacing between two contours corresponds to an energy change of $5 \cdot 10^{-5}$ a.u. with a dark background indicating regions where $E_{\mathrm{AP} 1 \mathrm{roG}}>E_{\mathrm{DOCI}}$. The thick line indicates the contour of $\Delta E=0$. 